\title{
Das Konzept der „,neutralen Gewalt“ - eine verfassungshistorische Überlegung zur Debatte um Präsidentialismus und Parlamentarismus ${ }^{1}$
}

\author{
Von Andreas Timmermann, Berlin
}

\section{Einführung}

Der gesellschaftswissenschaftliche Trend der 1980er Jahre, Entwicklungsdefizite und Instabilität vieler Staaten mit ihrer institutionellen Ausstattung zu erklären, hat sich auch auf die Lateinamerikaforschung ausgewirkt. Ausgehend von Juan Linz und seiner Schule ${ }^{2}$, haben zahlreiche Autoren unter dem Stichwort „failed presidencies“ spezifische Funktionsprobleme der Präsidialdemokratien identifiziert und ihnen gegenüber die Vorzüge parlamentarischer Demokratien hervorgehoben. Üblicherweise kennzeichnet das amerikanische Präsidialsystem, dass der Präsident für die Regierungsgeschäfte allein zuständig ist - worin es sich etwa vom semi-presidentiellen System in Europa unterscheidet. Auch wenn die Minister die Entscheidungen ausfertigen, sind sie „Gehilfen“ (Sekretäre), die der Präsident nach seinem Gutdünken ernennt und entlässt, während er selber nur durch ein Amtsenthebungsverfahren abzusetzen ist. Als Hauptproblem gilt in diesem Zusammenhang die für das Präsidialsystem charakteristische Gewaltentrennung zwischen dem Staats- und Regierungschef, dem Parlament und den obersten Gerichten. Die im Vergleich zu den parlamentarischen Systemen mangelnde Gewaltenverschränkung drücke sich besonders in der Unabhängigkeit des Präsidenten als Exekutive von der parlamentarischen Mehrheit aus: zum einen wegen des Prinzips der dualen Legitimität, wonach sich nicht nur das Parlament, sondern auch der Präsident auf eine unmittelbare Legimitation durch Wahlen berufen kann; zum anderen, weil dieser eine eigene, von der des Parlaments unabhängige Amtszeit hat, und der Verlust einer Parlamentsmehrheit kein Misstrauensvotum gegen den Präsidenten bedeutet $^{3}$. Zugleich wird darauf hingewiesen, dass gerade diejenigen Regierungssysteme in Lateinamerika, die als besonders stabil gelten können, am stärksten vom nordamerikani-

Der Beitrag ist die erweiterte Fassung eines Referats, das der Autor auf der Jahrestagung des Arbeitskreises für Überseeische Verfassungsvergleichung (AÜV) am 24. Juni 2006 in Osnabrück gehalten hat.

Juan Linz/Arturo Valenzuela, The Failure of Presidential Democracy, Baltimore 1994. schränkung in den lateinamerikanischen Präsidialdemokratien, in: Lateinamerika Analysen 7 (2004), S. $60 \mathrm{ff}, 78 \mathrm{ff}$. 
schen Muster des Gewaltentrennungsprinzips abweichen, so Chile, Costa Rica und Uruguay. 4

Der Nachteil dieses politisch-institutionellen Ansatzes der „failed presidencies“ liegt darin, dass er in einen mehr oder minder schematischen Systemvergleich mündet, der die jeweiligen historischen Bedingungen vernachlässigt. Damit kommen insbesondere die Zwischenstufen zu kurz, über die sich der heutige, keineswegs einheitliche Typus eines lateinamerikanischen Präsidentialismus entwickelt hat. Aus einer verfassungshistorischen Perspektive ist nämlich zu bezweifeln, dass sich in irgendeiner Phase der lateinamerikanischen Geschichte Gewaltentrennung im Sinne eines reinen, amerikanischen Präsidialsystems dem Prinzip der Gewaltenverschränkung im Sinne des klassischen, europäischen Parlamentarismus gegenüberstellen ließe. Seit dem 19. Jahrhundert haben die lateinamerikanischen Verfassungen immer wieder gerade mit Blick auf die Gewaltentrennung - ganz abgesehen vom Parteiensystem und vom Wahlrecht - Mischformen verankert. Es scheint ein Charakteristikum des lateinamerikanischen Konstitutionalismus zu sein, sich immer wieder europäischer Vorbilder zu bedienen und eben nicht nur dem Vorbild der Verfassung von Philadelphia aus dem Jahre 1787 zu folgen: sei es, um ein quasi monarchisches Staatsoberhaupt einzusetzen oder neben dem direkt gewählten Präsidenten einen Premierminister zu installieren und durch dessen Abhängigkeit vom Parlament die Exekutive gleichsam zu ,pparlamentarisieren"; sei es um der einheitlichen Exekutive eine in mehr als zwei Kammern aufgefächerte Legislative gegenüberzustellen. Vor diesem Hintergrund soll die vorliegende Untersuchung folgendes zeigen:

1. Die Staatengründungen in Lateinamerika waren von vornherein durch eine Suche nach Mischformen des Regierungssystems, also im Verhältnis zwischen Exekutive und Legislative gekennzeichnet, auch wenn sich dann vielfach das nordamerikanische Modell durchsetzte. Diese Suche nach vermittelnden Lösungen begann also nicht erst, wie der institutionelle Ansatz nahelegt, als sich im Laufe der Zeit Funktionsdefizite des Präsidialsystems nordamerikanischer Herkunft zeigten, sondern bereits in einer frühen Phase der Staatengründungen.

2. Dass der staatstheoretische Anteil Europas an diesen Mischlösungen so groß ist, hängt zum einen mit der Lehre des Schriftstellers und Politikers Henri Benjamin Constant de Rebecque (1767-1830) zusammen: Seine Figur der „,neutralen Gewalt“ (pouvoir neutre) hat im Süden des amerikanischen Kontinents einen viel stärkeren Einfluss als im Norden gewonnen. Zum anderen kam der Suche nach Mischlösungen zugute, dass zumindest in der Anfangsphase der Staatengründungen in Übersee fast überall Bestrebungen zur Einführung 
konstitutioneller Monarchien bestanden, auch wenn diese Staatsform nur in Brasilien während des 19. Jahrhunderts Bestand hatte.

Die Lehre Benjamin Constants bildet in dieser Hinsicht gleichsam ein Bindeglied zwischen dem europäischen und dem überseeischen Verfassungsdenken, was der Vergleich mit der konstitutionellen Monarchie in Spanien verdeutlicht. Aus historischer Sicht ist für jede Gegenüberstellung von Präsidentialismus und Parlamentarismus zu berücksichtigen, dass die parlamentarische Demokratie im westlichen Europa keineswegs unmittelbar auf das Ancien Régime beziehungsweise auf die sie ablösenden revolutionären Bewegungen folgte. Vielmehr entwickelte sich ihre heutige Form über die Zwischenstufe der konstitutionellen Monarchie, die man als ,verfassungshistorischen Normalfall der europäischen Verfassungsentwicklung im 19. Jahrhundert" bezeichnen kann ${ }^{5}$. Auf dieser Zwischenstufe spielte der liberale Gedanke einer vermittelnden, über den Gewalten stehenden ,,neutralen“ Funktion des Staatsoberhauptes eine Schlüsselrolle. Es hat die Rezeption dieses Gedankens in Übersee begünstigt, dass Constants Lehre dort zu einer Zeit bekannt wurde, als Teile Lateinamerikas zumindest formal noch im kolonialen Verbund mit der iberischen Halbinsel standen. Zugleich übte der spanische Konstitutionalismus, durch seine Vermittlung befördert auch der französische, danach weiterhin starken Einfluss auf die Staatslehre in Lateinamerika aus, wofür die Rezeption Constants nur ein Beispiel unter mehreren ist. ${ }^{6}$

\section{Der ,pouvoir neutre“ oder, ,pouvoir préservateur“bei Benjamin Constant}

Den Überlegungen Constants geht die Einsicht voraus, dass sowohl die Exekutive wie auch die Legislative ihre Macht missbrauchen können und daher beide Gewalten (oder Funktionen) wirksam zu beschränken seien. Denn zur Despotie führt aus seiner Sicht jede Allmacht, entweder der Individuen oder einer Versammlung ${ }^{7}$. Zugleich unterstellt Constant, dass für diese Beschränkung selbst eine sorgsame Trennung der Kompetenzbereiche und eine gegenseitige Kontrolle beider Gewalten keineswegs ausreichen, auch nicht durch Einbeziehung der Gerichte. Einerseits bestehe die Gefahr, dass sich diese Gewalten befeh-

Martin Kirsch, Monarch und Parlament im 19. Jahrhundert: der monarchische Konstitutionalismus als europäischer Verfassungstyp, Göttingen 1999, S. 24, 44 f; zustimmend: Hans-Christof Kraus, Monarchischer Konstitutionalismus. Zu einer neuen Deutung der deutschen und europäischen Verfassungsentwicklung im 19. Jahrhundert, in: Der Staat 43 (2004), S. 600.

M.w.N. zur ideen- und rechtgeschichtlichen Mittlerrolle Spaniens für Lateinamerika: Andreas Timmermann, ,Soziale Solidarität“ und Agrarreform im 20. Jahrhundert: zur Wirkung der Rechtsschule Léon Duguits - ein überseeischer Vergleich, in: Verfassung und Recht in Übersee 37 (2004), S. $168 \mathrm{ff}$.

Benjamin Constant, Principes de politique. Applicables à tous les gouvernements représentatifs et particulièrement à la constitution actuelle de la France (1815), 1. Kap., in: ders., Écrits politiques, Paris 1997, S. 321. 
den und sich niemand findet, der Eintracht zwischen ihnen stiftet. Andererseits könnten sie gemeinsame Sache machen, so dass sich niemand ihren Übergriffen zu widersetzen vermag. Der Schluss hieraus lautet: Um das Gemeinwesen entweder vor dem Bürgerkrieg zu bewahren, wenn sich der Streit zwischen Parlament und Regierung zuspitzt, oder aber vor der Despotie, wenn beide gemeinsam die natürlichen Rechte der Bürger verletzen, muss eine vermittelnde, von diesen verschiedene Instanz die verfassungsmäßige Ordnung und Freiheit schützen. So erklärt sich zugleich die Umschreibung dieser vermittelnden ,neutralen“ Gewalt (force, pouvoir neutre) als „,bewahrende“ Gewalt (force préservatrice, pouvoir préservateur). ${ }^{8}$

Ideengeschichtlich betrachtet, war der Gedanke Constants nicht ganz neu: Anklänge fanden sich bereits in der französischen Literatur des 19. Jahrhunderts, so in der Idealisierung des englischen Königs als ,,pouvoir mitoyen“, der zwischen Lords und Gemeinen vermittelte 9 Wie für die Theorie der Gewaltenteilung überhaupt, wirkte auch für diesen Gedanken die englische Verfassungspraxis als Vorbild. Dies betraf besonders die Rolle des Monarchen, wenn er, einem englischen Richter vergleichbar, quasi in letzter Instanz über die Einhaltung der Gesetze und der Verfassung wachen und die Individuen vor einem Machtmissbrauch der staatlichen Organe bewahren sollte. Die französischen Revolutionäre haben daran angeknüpft: In diesem Sinne sprach der zweimalige Präsident der Nationalversammlung, Stanislas de Clermont-Tonnerre (1747-1792) von einem ,pouvoir régulateur“ und meinte damit das Veto- und Gnadenrecht des Monarchen. Übertragen auf die Republik, erkannte die Konsulatsverfassung von 1799 nun einer Versammlung, dem „Sénat conservateur“, jene ausgleichende Funktion zu, wonach die Versammlung einerseits über den Gewalten stehen und andererseits das Gleichgewicht zwischen ihnen herstellen sollte. ${ }^{10}$

Aus diesen Voraussetzungen ergeben sich die Vorrechte des ,pouvoir neutre“, wie Constant ihn verstand: nämlich den Inhaber der exekutiven Gewalt abzusetzen und die Bildung einer neuen Regierung zu ermöglichen, aber auch das Parlament aufzulösen und somit Neuwahlen einzuleiten; ferner verfügt er über das Gnadenrecht; er kann die Exekutive auf Beanstandungen der Regierten aufmerksam machen und schließlich Verfassungsänderungen gutheißen ${ }^{11}$. Auch wenn diese Befugnisse, wie erwähnt, an die richterliche Gewalt erinnern, unterscheiden sie sich im Wirkungsbereich hiervon. Der ,pouvoir neutre“

Benjamin Constant, Principes de politique, 2. Kap., in: Écrits politiques (Anm. 7), S. 324; ferner Norbert Campagna, Benjamin Constant. Eine Einführung, Berlin 2003, S. 157.

Dazu Stephen Holmes, Benjamin Constant and the Making of Modern Liberalism, New Haven 1984, S. 144 f, mit Bezug auf Voltaire.

10 Lothar Gall, Benjamin Constant. Seine politische Ideenwelt und der deutsche Vormärz, Wiesbaden 1963, S. 168 ff; vgl. 2. Titel („Du Sénat conservateur“), Art. 15 ff Constitution du 22 frimaire an VIII. 
ist nicht wie der Richter an das Gesetz gebunden, sondern verfügt - allerdings nur innerhalb seines Aufgabenbereichs - über eine diskretionäre Macht (,autorité discrétionnaire“) im Sinne einer politisch unbeschränkten Vollmacht, die präzise weder durch das Gesetz noch durch Verfassungsnormen zu begrenzen ist. ${ }^{12}$

Besonders relevant für die spätere Rezeption, gerade in Übersee, ist die Frage nach der Staatsform: Offenbar ging Benjamin Constant davon aus, dass seine Lehre vom ,pouvoir neutre“ sowohl mit der Republik als auch mit der (konstitutionellen) Monarchie vereinbar sei. Hierfür spricht, dass sein Hauptaugenmerk der Begrenzung staatlicher Macht an sich galt. Diese bedeutete die Anerkennung der Volksouveränität und der Individualrechte, insbesondere der Religions-, Meinungs- und Eigentumsfreiheit, ferner die Verantwortlichkeit der Exekutive vor dem Gesetz sowie den Schutz vor staatlicher Willkür durch die Richter $^{13}$. Diese Bedingungen können aber beide Staatsformen gewährleisten ${ }^{14}$. Hinzu kommt ein biographischer Umstand: Constant unterstützte im Verlauf seines intellektuellen und politischen Wirkens sowohl die Republik wie auch die Monarchie, trat zeitweise für und gegen Napoleon ein. Im Grunde war er undogmatisch und konnte sich mit sehr verschiedenen Staats- und Regierungsformen arrangieren, solange diese dem Schutz der individuellen Freiheit dienten. In diesem Punkt dachte Constant allerdings dogmatisch. ${ }^{15}$

Die über den anderen Gewalten stehende, neutrale Gewalt muss daher nicht zwingend der König, sondern kann auch eine weitere Versammlung sein: Deren Mitglieder sollten nach den Vorstellungen Constants allerdings qualifizierende Voraussetzungen erfüllen, was Alter und Vermögen anbelangt, und nach Möglichkeit auf Lebenszeit gewählt sein, wodurch sie nicht nur größere Unabhängigkeit, sondern auch ein besonderes Renommée erlangten ${ }^{16}$. Vor allem diese republikanische Variante einer vermittelnden Gewalt, die zugleich aristokratische Tendenzen verrät, ist in Lateinamerika rezipiert worden.

Allerdings macht Constant keinen Hehl daraus, dass sich aus seiner Sicht der Monarch für diese Rolle besonders gut eigne: Zum einen war der König in fast allen europäischen Staaten „Verfassungswirklichkeit“, so dass keine neue Institution geschaffen werden musste, die sich die notwendige Autorität erst hätte erwerben müssen. Zum anderen symbolisierte

Norbert Campagna, Benjamin Constant (Anm. 8), S. 157 f; Florian Weber, Benjamin Constant und der liberale Verfassungsstaat. Politische Theorie nach der Französischen Revolution, Wiesbaden 2004, S. 278.

Benjamin Constant, Principes de politique, 1. Kap., in: Écrits politiques (Anm. 7), S. 317, $321 \mathrm{f}$.

Florian Weber, Benjamin Constant und der liberale Verfassungsstaat (Anm. 12), S. 255 f; i.d.S. auch Norbert Campagna, Benjamin Constant (Anm. 8), S. 158.

Woldemar Bach, Benjamin Constant und die Politik, 1767-1802. Eine psychologisch-historische Studie, Leipzig 1936, S. 51 f.

16

Stephen Holmes, Benjamin Constant and the Making of Modern Liberalism (Anm. 9), S. 145. 
am ehesten die Person des Königs die politische Einheit der Nation und verkörperte aufgrund der erblichen Stellung, die den politischen Tagesgeschäften enthoben war, Leidenschaftslosigkeit und Ruhe ${ }^{17}$. Diese „Neutralität“ des Königs betraf seine Person in doppelter Hinsicht: Einerseits verkörperte er die Unparteilichkeit des Staates insgesamt und stand als Person für die Möglichkeit zur öffentlichen Debatte überhaupt. Andererseits lag hierin auch eine Beschränkung seines Handlungsspielraums. Denn er durfte nicht seinem persönlichen Willen als dem maßgeblichen Kriterium folgen. Wenn also Constant den Monarchen rhetorisch emporhob, wurde dessen politischer Wille durch seine Funktion als Schiedsrichter gleichsam „neutralisiert“ “18. Dieser Gedanke stand auch hinter der Trennung der monarchischen (,,pouvoir royal“) von den ausführenden Befugnissen (,,pouvoir exécutif“) des Königs. Constant legte deshalb Wert auf die Unterscheidung zwischen ,auctoritas“ und ,potestas“, weil er in der historischen Verbindung der politischen Autorität mit der politischen Macht einen wesentlichen Grund dafür sah, dass die Macht die Autorität hatte korrumpieren können und die Krone in den Augen des Volkes die Legitimation verloren hatte, weiterhin die Gesamtheit der Nation zu vertreten. ${ }^{19}$

\section{Das Staatsoberhaupt als ,poder neutral y moderador“ in den spanischen Verfassungen des 19. Jahrhunderts}

Eine erste juristische Grundlage für den späteren Rekurs auf Constants Lehre vom,,pouvoir neutre" schuf die Verfassung von 1812: Nach den Erfahrungen des Absolutismus unternahm es erstmals in der neueren spanischen Geschichte die Konstituante von Cádiz, die strukturell als despotisch eingeschätzte und zur Willkür tendierende Herrschaft eines Einzelnen, des Monarchen, zu bändigen. Nicht im Vordergrund stand die Vorstellung, dass das Staatsoberhaupt zwischen verfeindeten Parteien und im Verhältnis der übrigen öffentlichen Gewalten schlichten müsse. Allenfalls das Parlament, als vom Volk gewählter Repräsentant der Nation, konnte eine solche vermittelnde oder überwachende, kontrollierende und erforderlichenfalls ahndende Funktion übernehmen. Der König sollte daher nicht über den Gewalten stehen und auch keine eigene Funktion neben jener des Kabinetts als Spitze der Exekutive ausüben. In diesem Sinne sprach die Verfassungskommission, einleitend zu ihrem Entwurf des Jahres 1811, vom König als dem „Vorsteher der Regierung und ersten

Das unterschied aus Constants Sicht die rechtmäßige monarchische Macht von der angemaßten Macht des Usurpators, Benjamin Constant, Über die Gewalt. Vom Geist der Eroberung und von der Anmaßung der Macht (De l'Esprit de conquête et de l'Usurpation dans leurs rapports avec la civilisation européenne, 1814), Stuttgart 1948, 2. Teil, 2. Kap., S. 60 f, 66. 
Amtsträger der Nation“ ${ }^{20}$. Wie in vielen Punkten folgte die spanische Konstituante hierin noch der französischen von 1791, die als erste die von verfassungsmäßigen Bindungen freie, absolute Stellung des Königs im Ancien Régime in eine abgeleitete, exekutive Funktion überführte, wonach konsequenterweise nicht mehr vom „König Frankreichs“, sondern allenfalls vom „König der Franzosen“ die Rede sein konnte ${ }^{21}$. Die Liberalen bestanden auf der ungeteilten, unveräußerlichen Souveränität der Nation, so dass auch der König lediglich abgeleitete Befugnisse haben durfte und als pouvoir constitué neben anderen zu gelten hatte. Die Verfassung sollte seine Befugnisse binden, nicht aber den König aus dem institutionellen Gefüge herauslösen. Gegen den Gedanken einer vermittelnden Gewalt sprach ferner auch die damals herrschende Vorstellung von einem harmonischen Ausgleich und Gleichgewicht der öffentlichen Funktionen, weshalb man es 1812 nicht für erforderlich hielt, eine der beteiligten Institutionen über die anderen emporzuheben. Mit der Sonderstellung einer weiteren, vierten Gewalt wäre das Eingeständnis verbunden gewesen, dass die übrigen staatlichen Funktionen sich weder in einem organischen Gleichgewicht befanden, noch ein institutionelles Gleichgewicht zwischen ihnen herzustellen war.

Nachdem im Jahr 1820 die spanische Übersetzung seiner „Principes de politique. Applicables à tous les gouvernements représentatifs“ erschienen war $^{22}$, verbreiteten sich die politischen Ideen Benjamin Constants über kleinere, gebildete Zirkel hinaus in Spanien und in Lateinamerika. Die Schrift wurde in Spanien zum Standardwerk liberaler Theorie und zur Referenz für alle diejenigen, die den Absolutismus der Bourbonen oder den Konservatismus der Verfassungsgegner ablehnten ${ }^{23}$. Eine Vorreiterrolle in der Anwendung jenes weltanschaulich überhöhten Konzepts, welches den König als Staatsoberhaupt über den Kampf der Parteien erhaben macht, übernahm die französische Monarchie im Zeitalter der Restauration. Im juste-milieu-Denken Frankreichs belebte sich jene, viel ältere Idealisierung des Machthabers wieder, nun des Bürgerkönigs, der unter den veränderten Bedingungen des Parlamentarismus ,herrscht, aber nicht regiert““24. Über Legislative und Exekutive herausgehoben, wurde er zum „pouvoir neutre“, zur neutralen Gewalt, „ein unsichtbares, alle Gegensätze und Reibungen zwischen den verschiedenen staatlichen Tätigkeiten und

„El Rey, como jefe del Gobierno y primer magistrado de la nación“, Agustín de Argüelles, Discurso preliminar a la Constitución de 1812 (1811), Madrid 1989, 1. Teil, S. 89.

Federico Suárez, Sobre las raices de las reformas de las Cortes de Cádiz, in: Revista de Estudios Políticos 126 a.F. (1962), S. 57. María Luisa Sánchez-Mejía, Benjamin Constant y la construcción del liberalismo posrevolucionario, Madrid 1992, S. 21.

24 „Le roi règne, mais il ne gouverne pas", Carl Schmitt, Politische Theologie II: die Legende von der Erledigung jeder politischen Theologie, Berlin 1970, S. 52 ff. 
Funktionen ausgleichendes, temperierendes und moderierendes Moment, ein invisible modérateur". 25

In Spanien wurde der Krone mit der Verfassung von 1845 und danach im Zuge der Restauration des Jahres 1875 eine schlichtende Funktion zugewiesen, nämlich zwischen der Exekutive, dem Rat der Minister (Consejo de Ministros), und dem Parlament, den Cortes. Man kann von einer vierten, vermittelnden Gewalt (poder moderador) sprechen, die im Grunde über den anderen stand. Das Übergewicht des Monarchen beruhte stärker noch als auf der Beteiligung an der Gesetzgebung auf seinen Rechten zur Auflösung der Cortes sowie zur Ernennung und Abberufung der Minister ${ }^{26}$. In Spanien hatte die Rezeption dieses Gedankens einen realen politischen Hintergrund: Nach den schweren Auseinandersetzungen zwischen Verfassungsbefürwortern und Anhängern des modernen Parlamentarismus auf der einen und den Anhängern des Absolutismus, mindestens aber eines monarchischen Prinzips, auf der anderen Seite, nach der Abspaltung und zunehmenden Radikalisierung neuer progressiver Gruppierungen und auf dem Höhepunkt der Karlistenkriege sprach viel dafür, einen über die Auseinandersetzungen der Funktionsträger und Parteien erhabenen königlichen Schlichter zu verankern. Letztlich war diese Konzeption ein Kompromiss: Denn einerseits konnte der Monarch nicht mehr selber der Souverän mit konstituierender Gewalt (poder soberano y constituyente) sein. Andererseits sollte er auch nicht eine wie die übrigen staatlichen Funktionen bloß abgeleitete und von vornherein beschränkte Gewalt (poder constituido y limitado) bleiben, weshalb man ihn zu einer über die anderen herausgehobenen, neutralen oder vermittelnden Gewalt (poder neutral y moderador) erklärte. ${ }^{27}$

Auch wenn man die zuvor erwähnten Einschränkungen berücksichtigt, gab bereits 1812 die Verfassung von Cádiz einen ersten wichtigen Hinweis auf die später anerkannte Stellung des Monarchen als eigenständige, vermittelnde Gewalt. Art.168 erklärt die Person des Königs für heilig und unantastbar, ohne einer juristischen oder politischen Verantwortlichkeit unterworfen zu sein ${ }^{28}$. Diese Formulierung findet sich danach in den Verfassungen von 1837 (Art. 44), 1845 (Art. 42) und 1869 (Art. 67). Schließlich hat man sie auch in Art. 48 der Verfassung von 1876 aufgenommen, wenn auch ohne die Klarstellung, dass der Monarch selber nicht verantwortlich ist, während die Verantwortlichkeit der Minister

Walther L. Bernecker/Horst Pietschmann, Geschichte Spaniens: von der frühen Neuzeit bis zur Gegenwart, 2. Aufl., Stuttgart 1997, S. 224; siehe Art. 26 bzw. Art. 45 Abs. 10 der Verfassung von 1845 sowie Art. 32 bzw. 54 Abs. 9 der Verfassung von 1876.

27

Pedro de Vega, El poder moderador, in: Revista de Estudios Políticos 116 n.F. (2002), S. 11.

28

„La persona del Rey es sagrada e inviolable, y no está sujeta a responsabilidad“, in: Jorge de Esteban, Las Constituciones de España, 2. Aufl., Madrid 1988, S. 69; ferner die Verfassungskommission: Agustín de Argüelles, Discurso preliminar a la Constitución de 1812, 1. Teil (Anm. 20), S. 90. 
weiterhin ausdrücklich erwähnt wird (Art. 49). Die Unverletzlichkeit und Nichtverantwortlichkeit des Monarchen und Staatschefs bilden die verfassungsrechtliche Basis des poder moderador, da ansonsten alle Amtsträger, auch die Minister, verantwortlich sind. Der König mag als Person und Amtsträger fehlgehen. Aber er ist weder juristisch noch politisch zur Rechenschaft zu ziehen. Ansonsten müsste man eine höhere, souveräne Instanz annehmen, die befugt wäre, eine solche Rechenschaft einzufordern, wie sie die Verfassungsväter von Cádiz noch für die Nation reklamierten ${ }^{29}$. Dort, wo eine vergleichbare Klausel der Unverletzlichkeit und Nichtverantwortlichkeit des Staatschefs fehlt, haben sich die Verfassungen für die Alternative entschieden: Es handelt sich dann nicht um den Chef des Staates, sondern um den der Exekutive, also den Regierungschef oder Ministerpräsidenten.

Allerdings deutete sich mit dem Grundsatz, dass der König selber nicht zur Rechenschaft gezogen werden konnte, wohl aber andere Mitglieder der Exekutive, bereits eine entscheidende Schwächung seiner Stellung an, die in Spanien ebenso wie in den übrigen konstitutionellen Monarchien Westeuropas den Übergang zur parlamentarischen Monarchie vorbereitete: In dem Maße, in dem der König über die übrigen Funktionsträger herausgehoben war, nahm er auch nicht mehr entscheidend an den Regierungsgeschäften teil. Und wenn der König selber nicht verantwortlich war, durfte er auch keine Staatsakte aus eigener Verantwortung durchführen. Denn dies hätte dem Grundsatz der Oberherrschaft des Rechts widersprochen ${ }^{30}$. Soweit nach dem Vorbild der Vereinigten Staaten die spanischamerikanischen Regierungssysteme im 19. Jahrhundert in der Person des Präsidenten die Funktionen des Staats- und Regierungschefs miteinander verbunden ließen, vermieden sie diese Folge. Auch konnte dieser zumindest über das Institut der Präsidentenanklage zur Verantwortung gezogen werden. Aus dem Umstand, daß nach rechtsstaatlichen Grundsätzen formell wie materiell für alle hoheitlichen Entscheidungen irgendjemand verantwortlich sein muß, ergibt sich also eine wesentliche Bindung des poder moderador. Während der Präsident seine Staatsakte allein unterzeichnet, muß ersterer seine Verfügungen den zuständigen Ministern zur Gegenzeichnung vorlegen, da diese - im Unterschied zum König - in ihrem jeweiligen Ressort den Cortes verantwortlich sind. Dieser Vorbehalt der Gegenzeichnung in Art. 225 der Verfassung von Cádiz findet sich danach auch in den Verfassungen von 1837 (Art. 61), 1845 (Art. 64), 1869 (Art. 87) und 1876 (Art. 49). Die Gegenzeichnung durch den zuständigen Minister bedeutete den schriftlichen Nachweis für die Übernahme der Verantwortung dergestalt, dass sich die Exekutive eine Verfügung des Monarchen zur Richtlinie des eigenen Regierungshandelns machte. In dieser Verantwortungsübernahme liegt ein wesentliches Merkmal eines Ministerrats oder Kabinetts. Dagegen ist von einem solchen in den (reinen) Präsidialsystemen Nord- und Südamerikas nicht die Rede, da die 
Sekretäre (secretaries, secretarios) den Präsidenten zwar beraten, er aber selbst den Ausschlag gibt. ${ }^{31}$

Das Attribut „heilig“ (sagrada) bezüglich der Person des Königs knüpfte an die religiös überhöhte Stellung des Patriarchen und Oberhauptes im Volksstaat an. Er war entweder selber göttlichen Ursprungs oder leitete seine Befugnisse von einer göttlichen Verleihung $\mathrm{ab}$ - eine Vorstellung, die sich im Ancien Régime erhalten hatte ${ }^{32}$. Zum Gottesgnadentum gehörte auf dem Höhepunkt seiner theoretischen Entwicklung im 17. Jahrhundert der Glaube an ein besonderes, von menschlicher Einwirkung unabhängiges und unveräußerliches Regierungsrecht des Monarchen, und zwar durch Geburt (Legitimitätsprinzip) sowie durch göttliche Weihe (sakraler Charakter) ${ }^{33}$. Im weltlichen Verfassungsstaat des 19. Jahrhunderts spiegelte sich dieser Gedanke nurmehr in der Verbindung des Königs mit der Nation wider. Er repräsentierte, ja verkörperte die Einheit der Nation, deren Integrität wie die seine unantastbar war und deren Achtung den Respekt gegenüber dem Monarchen implizierte. Seine Entsprechung findet dieser Ansatz in der republikanischen Staatsform, sobald dem Präsidenten als Chef des Staates die Rolle eines „Garanten nationaler Unabhängigkeit“" und ,territorialer Integrität“ übertragen wird. ${ }^{34}$

Dieses Verständnis ging über das Konzept der ersten spanischen Konstituante von 1812 hinaus, demzufolge nur die Cortes und die in ihr versammelten Delegierten die Nation vertreten konnten. Auch tatsächlich bedurften sie nicht der Vermittlung eines Monarchen, dessen Unfähigkeit hierzu während seines Exils in Frankreich (1808-1814) offenkundig war. Vor diesem Hintergrund verbot es sich von selbst, dem König das alleinige Recht nicht nur zur Einberufung, sondern auch zur Auflösung des Parlamentes zu gewähren. Seit Mitte des 19. Jahrhunderts jedoch galt eben dieses Recht als Inbegriff der ausgleichenden, schlichtenden Funktion des poder moderador. Der Monarch verfügte die Auflösung der Cortes und das Ende der Abgeordnetenmandate, um die Wahl eines neuen Parlaments zu

Karl Löwenstein, Der Staatspräsident. Eine rechtsvergleichende Studie, in: ders., Beiträge zur Staatssoziologie, Tübingen 1961, S. 338, Ernst Fraenkel, Das amerikanische Regierungssystem, in: ders., Gesammelte Schriften, Bd. 4 (Anm. 30), S. 744 f.

Tilman Struve, Die Begründung monarchischer Herrschaft in der politischen Theorie des Mittelalters, in: Zeitschrift für Historische Forschung 23 (1996), S. 290 ff, 322 f; für Frankreich: Bertrand de Jouvenel, Über die Souveränität: Auf der Suche nach dem Gemeinwohl, Neuwied 1963, S. 60 ff; für England kritisch bereits: Algernon Sidney, Discourses concerning government (1698), Indianapolis 1990, 3. Kap., 28. Abschn., S. 478, mit Bezug auf die Schrift Robert Filmers, Patriarcha or the natural power of kings (1680).

Fritz Kern, Gottesgnadentum und Widerstandsrecht im frühen Mittelalter: zur Entwicklungsgeschichte der Monarchie, 2. Aufl., Darmstadt 1954, S. 3. 
ermöglichen ${ }^{35}$, die nicht nur ein besseres Zusammenwirken von Exekutive und Legislative gewährleisten, sondern auch den veränderten politischen Anforderungen und den Erwartungen der Öffentlichkeit entsprechen sollte. Ihm selber und nicht dem Parlament oblag es, das Volk zur Abstimmung aufzurufen, worin sich der Anteil des Monarchen an der Souveränität und der Unterschied zum Grundsatz der nationalen Repräsentation im Sinne der Verfassung von 1812 ausdrückte. Nur diejenige Instanz, die über Beginn und Ende der Legislatur entschied, vermochte Verfassungskonflikte auszugleichen und zu beenden. Eine ähnliche Überlegung stellt der Verfassungsgeber auch heute noch dort an, wo er den Staatschef mit der Aufsicht über das Zusammenwirken der öffentlichen Gewalten beauftragt und ihn zur Auflösung des Parlaments ermächtigt. ${ }^{36}$

\section{Das Konzept des ,poder moderador“ in Lateinamerika während des 19. Jahrhunderts}

In Lateinamerika findet sich Benjamin Constants Konzept des ,pouvoir neutre“ in beiden Staatsformen wieder, und zwar nicht nur in der häufigeren republikanischen Variante, sondern auch in einer monarchischen. Letztere hatte im 19. Jahrhundert großen Einfluss auf die brasilianische Verfassungsgeschichte. Allerdings gab es auch in den anderen Staaten, die sich im Verlauf der Unabhängigkeitsbewegung schließlich als Republiken konstituierten, vielfach starke monarchische Bestrebungen. Sie orientierten sich an den konstitutionellen Monarchien in Europa und zielten darauf ab, dem König eine Stellung einzuräumen, die der Vorstellung eines vermittelnden, über den klassischen Funktionsträgern angesiedelten ,Streitschlichters“ nahe kam.

\section{a. Konstitutionell-monarchische Ansätze}

Im Gebiet des ehemaligen Vizekönigreichs Río de la Plata verfolgten vor allem Manuel Belgrano (1770-1820) und seine Parteigänger einen konstitutionell-monarchischen Ansatz. Einige von ihnen, so Bernardino Rivadavia (1780-1845) waren gut vertraut mit den Ideen Benjamins Constants. Diese waren in den Salons von Buenos Aires ein beliebter Gesprächsgegenstand, zumal sie dem Zeitgeist entsprachen, der einem romantischen, gleichwohl elitären Liberalismus zuneigte ${ }^{37}$. Das aus Sicht der Liberalen abschreckende Beispiel der Französischen Revolution und die Furcht vor einer egalitär-demokratischen Herrschaft der Masse bestärkten sie in der Überzeugung, Argentinien müsse dem englischen Vorbild

Siehe Art. 32 S. 2 der Verfassung von 1876, der die erneute Einberufung der aufgelösten Kammer(n) (Senat und/oder Abgeordnetenhaus) binnen dreier Monate vorschreibt.

36 So gemäß Art. 5 Abs. 1, Art. 12 Abs. 1 der französischen Verfassung von 1958.

37

Otto Carlos Stoetzer, Benjamin Constant and the doctrinaire liberal influence in Hispanic America, Pittsburgh 1977, S. 11 ff. 
mit einem wirkungsvollen Balancesystem und einem harmonischen Ausgleich intermediärer Instanzen folgen. Daher schlug Belgrano in seinen wechselnden monarchischen Konzepten mal die Proklamation eines Mitglieds des spanischen Königshauses, mal die Wahl eines indigenen amerikanischen Königs (Inca) zum Oberhaupt einer gemäßigten, konstitutionellen Monarchie (monarquía templada) vor ${ }^{38}$. Auch in Peru und besonders in Hochperu (Alto Perú), das zum Vizekönigreich Río de la Plata gehörte, hatte sich die Vorstellung einer legitimen Inkaherrschaft erhalten. Sie war durch den Indianeraufstand von 1780/1781 unter Führung des José Gabriel Condorcanquí (Tupac Amarú II., 1743-1781) wiederbelebt worden und fußte auf der Überzeugung, dass den Nachfolgern der Inkas das Recht zustand, einen eingeborenen König zu designieren und ihm zu huldigen, nachdem der spanische Monarch die Krone des letzten Inkaherrschers usurpiert habe und sie daher nun auf die legitimen Erben rückzuübertragen sei ${ }^{39}$. Mit dem bekanntesten dieser Verfassungsentwürfe sollte eine „,neue Monarchie Südamerikas unter dem Namen Vereinigtes Königreich Rió de la Plata, Perú und Chile“ entstehen ${ }^{40}$. In dem Entwurf findet sich ein Plädoyer für ein Zweikammersystem nach englischem Vorbild, mit einer gesonderten Vertretung des Erbadels und der hohen Geistlichkeit im Oberhaus (Art. 7 Abs. 2) und des Volkes im Unterhaus (Art. 8). Dem König, dem neben der Legislative das Recht zur Gesetzesinitiative bleibt, war die Einberufung und Auflösung des Parlaments vorbehalten (Art. 9), wie es auch den Vorstellungen Constants und den folgenden spanischen Verfassungen des 19. Jahrhunderts entsprach. Auf dem Kongress von Tucumán (1816) neigte eine Mehrheit der Abgeordneten einer Monarchie zu; auch in der Folgezeit vertraten die Parteigänger dieser Staatsform ihre Position mit großem Nachdruck, bis sich in den zwanziger Jahren diese Frage zugunsten der Republik entschieden hatte ${ }^{41}$. Auch in Peru unterlagen die monarchischen Konzepte. Schließlich verzichteten die Kreolen darauf, unter Berufung auf die vermeintliche Legitimität der Inkaherrschaft eine gemäßigte Monarchie zu konstituieren. Dies schloss allerdings nicht aus, dass sie an die alten Rechte der Inkas appellierten, um

Aus biographischer Sicht: Jorge Newton, Belgrano. Una vida ejemplar, Buenos Aires 1970, S. 163 ff, 177 ff; zu den verschiedenen Konzepten für eine konstitutionelle Monarchie am Río de la Plata: Reinhold Blaurock, Monarchische Ideen und Initiativen am Río de la Plata zu Beginn der Unabhängigkeitsepoche (1808-1816), Frankfurt/M. 1998; Rubén Darío Salas, Los proyectos monárquicos en el proceso de la independencia argentina (1810-1820), in: Ibero-Amerikanisches Archiv 15 (1989), S. 193 ff.

Dazu Scarlett O`Phelan Godoy, El mito de la „Independencia concedida”: Los programas políticos del siglo XVIII y del temprano XIX en el Perú y Alto Perú (1730-1814), in: Inge Buisson / Günter Kahle / Hans-Joachim König / Horst Pietschmann, Problemas de la formación del Estado y de la nación en Hispanoamérica, Köln 1984, S. 66 ff.

So Art. 1 Proyecto de Constitución Monárquica de Rivadavia y Belgrano (1815), in: Arturo Enrique Sampay, Las Constituciones de la Argentina (1810-1972), Buenos Aires 1975, S. 207 ff.

41

Matías E. Suárez, Historia de las instituciones políticas argentinas 1810-1943, 2. Aufl., Buenos Aires 1974; S. 100; José Rafael López Rosas, Historia constitucional argentina, 5. Aufl., Buenos Aires 2002, S. 251. 
angesichts lokaler Erhebungen die Moral der Truppen zu stärken und auch die Masse der Eingeborenen zu erreichen. ${ }^{42}$

Infolge des Siegeszugs der republikanischen Staatsform überall in Lateinamerika ist heute in den Hintergrund getreten, dass auch andernorts maßgebliche Protagonisten der frühen Unabhängigkeitsbewegung zunächst monarchischen Konzepten zuneigten: In Neugranada verankerte zumindest kurzzeitig die erste Verfassung Cundinamarcas (1811) eine konstitutionelle Monarchie, ,,in der eine ständige nationale Repräsentation die Gewalt des Königs mäßigt “" ${ }^{43}$, bis sich wenig später die zweite Verfassung Cundinamarcas (1812) auf die Staatsform der Republik festlegte ${ }^{44}$. Als Mexiko seine Unabhängigkeit erlangte, ließ sich dort Augustín de Iturbide für eine kurze Regentschaft zum Kaiser (1822-1823) ausrufen. Ansätze gab es sogar in Venezuela, dem ersten Land, das sich für die republikanische Staatsform entschied. So hat der Rechtsgelehrte und Diplomat Andrés Bello (1781-1865) eine konstitutionelle Monarchie befürwortet, worin ihn ein längerer Englandaufenthalt und die dort erworbenen verfassungsrechtlichen Kenntnisse beeinflussten. Hieran knüpfte er in seinen späteren Stellungnahmen an, als es um die Ausarbeitung einer neuen chilenischen Verfassung (1831-1833) ging. ${ }^{45}$

\section{b. Die monarchische Variante: Benjamin Constants Rezeption in Brasilien}

Einen besonders starken Widerhall hatte Benjamin Constants Konzept einer neutralen oder vermitttelnden Gewalt in Brasilien, was auf verschiedene Umstände zurückzuführen ist: Sowohl in staatsrechtlicher als auch in dynastischer Hinsicht kennzeichnet die brasilianische Emanzipation eine gewisse Kontinuität mit der Kolonialgeschichte. Noch vor seiner Unabhängigkeit, im Jahr 1815, erhielt Brasilien gemeinsam mit Portugal den Status einer Monarchie (,reino unido“). Und anders als in den spanischamerikanischen Provinzen übertrug König João VI. selber seinem Thronerben die Regentschaft, worauf dieser eigenmächtig die Unabhängigkeit Brasiliens (1822) vom Mutterland erklärte und sich als Pedro I. zum Kaiser ausrufen ließ. Mit der dynastischen Verbindung blieb auch der geistige Einfluss Westeuropas ungewöhnlich stark, zumal der portugiesische Hof bis zum Vorabend der Unabhängigkeit in Übersee residierte (1808-1821) und mit zahlreichen Schulen, Aka-

Scarlett O`Phelan Godoy, El mito de la „Independencia concedida”, in: Problemas de la formación del Estado y de la nación en Hispanoamérica (Anm. 39), S. 70 f.

43

Constitución de Cundinamarca vom 30. 3. 1811, 1. Titel, Art. 4: „La Monarquía de esta provincia será constitucional, moderando el poder del Rey una Representación Nacional permanente", in: Diego Uribe Vargas, Las Constituciones de Colombia, Bd. 2: Textos 1810-1876, 2. Aufl., Madrid 1985, S. 351; ebenso auch 3. Titel, Art. 1 (ebda., S. 354).

Constitución de la República de Cundinamarca vom 18. 7. 1812, abgedruckt in: Diego Uribe Vargas, Las Constituciones de Colombia, Bd. 2 (Anm. 43), S. 581 ff.

45

Iván Jaksić, Andrés Bello: Scholarship and Nation-Building in Nineteenth-Century Latin America, Cambridge 2001, S. 44 ff. bzw. S. 103 f. 
demien und Kultureinrichtungen zur Verbreitung der neueren Ideen beitrug ${ }^{46}$. In einem spezifisch brasilianischen Eklektizismus verbanden sich die liberalen und die konservativrestaurativen Tendenzen dieser Zeit, die sich auch in der ersten brasilianischen „Verfassung“" von 1824 niederschlugen. Diese war nach dem Vorbild der französischen Charte Ludwigs XVIII. (1814) eigentlich ein Oktroi, den Pedro I. gnadenhalber gewährte, sich darin jedoch einer konstitutionell-monarchischen Ordnung unterwarf ${ }^{47}$. Art. 98 bezeichnete den Monarchen als „vermittelnde Gewalt“ und bezog sich darin unmittelbar auf Constants „Principes de politique“. Der Monarch hatte als Staats- und Regierungschef erheblichen Anteil an der exekutiven Gewalt, für deren Ausübung er nicht der Zustimmung seiner Minister bedurfte. Er war befugt, die Richter zu suspendieren und die Entscheidungen der Versammlungen in den Provinzen aufzuheben. Ihm oblagen das Gnadenrecht und die Auflösung des Abgeordnetenhauses, ferner Kriegserklärung und Friedenschluss sowie die Verhandlungen mit auswärtigen Mächten. Diese Aufzählung verdeutlicht, dass die Befugnisse des brasilianischen Kaisers umfassender waren, als sie dem ,pouvoir neutre“ nach dem Verständnis Benjamin Constants zustanden. Weder ist von einer Trennung der monarchischen Autorität von der eigentlichen exekutiven Gewalt zu sprechen, auf die Constant so großen Wert gelegt hatte, noch vom erwähnten juste-milieu-Ideal des Bürgerkönigs, der unter den Bedingungen des Parlamentarismus „,herrscht, aber nicht regiert“ ${ }^{48}$. Dem Anspruch nach sollte jedoch auch hier, allerdings unter anderen, lateinamerikanischen Verhältnissen, der Monarch eine stabilisierende und ausgleichende Funktion wahrnehmen; selbst wenn diese Rolle viel stärker im Sinne einer autoritären Führung und persönlichen Herrschaft aufgefasst und so insbesondere durch den Nachfolger des ersten Kaisers, Pedro II., ausgefüllt wurde.

\section{c. Bolivien und Peru: Das Konzept „vermittelnder Gewalten“ bei Simón Bolívar}

Eine dem brasilianischen Beispiel vergleichbare Umdeutung des „pouvoir neutre“ nahm auch Simón Bolívar (1783-1830) vor, als er in seinen späteren „Verfassungen“ auf Benjamin Constant rekurrierte ${ }^{49}$. In den Verfassungen Venezuelas von 1819 und Großkolum-

E. Bradford Burns, Nationalism in Brazil. A historical survey, New York 1968, S. 27; A.B. Cotrim Neto, O sistema constitucional brasileiro e a constituição de 1988, Rio de Janeiro c.a. 1988, S. 3 f.

47

Maßgeblichen Einfluss hatten auch die spanische Verfassung von 1812 und die portugiesische von 1822, Otto Carlos Stoetzer, Benjamin Constant and the doctrinaire liberal influence in Hispanic America (Anm. 37), S. 18.

Florian Weber, Benjamin Constant und der liberale Verfassungsstaat (Anm. 12), S. 17; Otto Carlos Stoetzer, Benjamin Constant and the doctrinaire liberal influence in Hispanic America (Anm. 37), S. 20. 
biens von 1821 hatte er noch eine stärkere Position der Exekutive gegenüber der Legislative in Friedenszeiten vermisst. Erst die durch ihn oktroyierten „Verfassungstexte“ von Bolivien und Peru (1826) korrigierten diesen vermeintlichen Mangel und entsprachen jenem (autoritären) Ansatz, den Bolívar allein als praktikabel für seine Regierung im nördlichen Südamerika ansah. ${ }^{50}$

Nach der endgültigen Befreiung Hochperus (Boliviens) wirkte er dort auf die Verabschiedung einer von ihm entworfenen „bolivarischen Verfassung“ (Constitución Bolivariana) hin, die er im gleichen Jahr auch für Peru oktroyierte ${ }^{51}$. Dieser Text entfernte sich am weitesten unter den frühen spanischamerikanischen Verfassungstypen vom liberalen spanischen Modell des Jahres 1812. Er hat aber auch mit der nordamerikanischen Verfassung von 1787 wenig gemein, sondern lässt wegen der überragenden Stellung der Exekutive am ehesten Parallelen zum napoleonischen Verfassungstyp erkennen: Die Präsidentschaft der Republik wird Simón Bolívar auf Lebenszeit übertragen (Abs. 5 und Art. 1 der Präambel) ${ }^{52}$. Als Ergänzung und Gegengewicht zu den klassischen drei Gewalten fungiert eine vierte, die „Wählergewalt“ (Poder Electoral, Art. 9, Art. 20 ff). Sie bestand aus Wahlkörpern, die in den Provinzen gewählt wurden, und Kandidaten für die Parlamentskammern sowie für die Posten der Präfekten, Gouverneure, Bürgermeister und Friedensrichter präsentierten. Zwar ging die Institution des „Poder Electoral“ auf die Verfassungstexte Napoleons der Jahre VIII und X zurück. Doch bezog sich Bolívar mit der Einrichtung einer vierten Gewalt als solcher auf den ,pouvoir neutre“ Benjamin Constants, der Konsequenzen aus den Fehlentwicklungen der Französischen Revolution hatte ziehen wollen, worauf Napoleon und nach ihm Simón Bolívar die neutrale Gewalt Constants in die Wählergewalt umdeuteten. $^{53}$

Eine weitere Institution leitete Bolívar aus Constants Konzept ab: nämlich die sog. „Moralische Gewalt“ (Poder Moral), eine aus „Zensoren“ zusammengesetzte Form der Laieninquisition, die gemeinsam mit „Tribunen“ und „Senatoren“ die Legislative bildete. Auch die Zensorenkammer orientierte sich am napoleonischen Vorbild, insofern die Zensoren

Constitución para la República Peruana (Denominada Constitución Vitalicia de Bolívar) vom 30.11.1826, in: Cámera de Diputados, Constituciones políticas del Perú 1821-1919, Lima 1922, S. $73 \mathrm{ff}$.

Den Vorschlag, eine Person auf Lebenszeit als ,elective monarch“ mit der exekutiven Gewalt zu betrauen, hatte (erfolglos) bereits Alexander Hamilton im Juni 1787 dem verfassunggebenden Konvent von Philadelphia unterbreitet; dazu Harald von Bose, Republik und Mischverfassung zur Staatsformenlehre der Federalist Papers, Frankfurt/M. 1989, S. 79.

Zur Begründung: Simón Bolívar, Discurso del Libertador al Congreso Constituyente de Bolivia (Lima, 25.5.1826), in: ders., Discursos, proclamas y epistolario político, Madrid 1975, S. 300; zum Einfluss der napoleonischen Konsulatsverfassung: Gerhard Masur, Simón Bolívar und die Befreiung Südamerikas, Konstanz 1949, S. 525. 
Bolívars wie die Senatoren Napoleons auf Lebenszeit ernannt wurden (Art. 15 S. 1 Verfassung von 1799). Und wie die bolivarische Zensorenkammer hatte auch der französische Senat die Funktion einer „republikanischen Inquisition“ übernommen, die die Gesetze und die öffentliche Moral überwachen sollte ${ }^{54}$. Die Ergänzung um eine neue vierte Gewalt, den Poder Moral, hatte Bolívar erstmals dem Kongress von Angostura in Venezuela (1819) vorgeschlagen $^{55}$, der es allerdings bei der klassischen Dreiteilung der Gewalten (oder Funktionen) beließ. Wie bereits die Einrichtung der Wahlkörper bezweckte diese Aufteilung der Legislative in drei konkurrierende Kammern (Cámera de Tribunos, Cámera de Senadores, Cámera de Censores, Art. 27 ff) eine gegenseitige Hemmung und damit letztlich die Schwächung des Repräsentivsystems. Bolívar ging es weniger um eine Verbesserung des Balancesystems, das er für eine Quelle fortgesetzter Streitigkeiten hielt, als vielmehr darum, die Alleinstellung des Präsidenten als Garanten des Allgemeinwohls zu festigen. Um diese oberste, dauerhaft durch nur eine Person auszuübende Gewalt sollten sich die übrigen Funktionen des Staates, ja eigentlich alle Amtsträger und Bürger, wie Gestirne um einen Fixstern bewegen. Diese Stellung des Präsidenten hielt Bolívar nicht nur für einen notwendigen Bestandteil in hierarchischen Systemen überhaupt. Er sah nach seinen Erfahrungen darin auch das einzige, durchaus demokratische Mittel, um die lateinamerikanischen Völker überhaupt regieren zu können.

Das Konzept Bolívars ist also nicht zu trennen von seinem Tugendverständnis. Er hielt die republikanische Gemeinschaft infolge ihrer inneren Zerrissenheit für so gefährdet, dass die Tugend ihrer Bürger mit staatlicher Autorität durchgesetzt werden müsse, auch wenn für diese Autorität durchaus institutionelle Schranken vorzusehen waren. Der Umstand, dass Bolívar eine immer stärkere Exekutive forderte und schließlich eine Präsidentschaft auf Lebenszeit durchsetzte, lässt sich damit erklären, dass er im Verlaufe des Emanzipationsprozesses skeptischer gegenüber der Selbstordnungsfähigkeit der Gesellschaft wurde ${ }^{56}$. Allerdings verwässerte Bolívars Konzept der vermittelnden Gewalten noch stärker als das brasilianische den Ausgangsgedanken Benjamin Constants, insofern Bolívar sich entschieden gegen einen Vorrang der Legislative wandte.

Otto Carlos Stoetzer, Bolívar und Europa (1783-1830), in: Wilhelm Stegmann, Simón Bolívar: Persönlichkeit und Wirkung, Berlin 1984, S. 67, 69.

Proyecto de Constitución para la República de Venezuela, formado por el Jefe Supremo y presentado al Segundo Congreso Constituyente para su examen, Apéndice a la Constitución relativo al Poder Moral, abgedruckt in: Pedro Grases, El Libertador y la Constitución de Angostura de 1819, Caracas 1970, S. 197 ff. proclamas y epistolario político (Anm. 53), S. 303, 306; ferner Ulrich K. Preuss, Der Feldherr als Verfassunggeber, in: Simón Bolívar, Rede von Angostura am 15. Februar 1819, Hamburg 1995, S. 98. 
Hierin lag auch der Hauptvorwurf der Gegner Bolívars und der von ihm oktroyierten Staatsordnung, die in Peru bereits 1827 wieder aufgehoben wurde. Der verfassunggebende Kongress Perus entschied sich für die Rückkehr zur Aufteilung in drei Staatsfunktionen (oder Gewalten), wobei die gesetzgebenden Befugnisse des Kongresses nun konsequenter als noch in der ersten Verfassung von 1823 auf zwei Kammern, das Abgeordnetenhaus (Cámera de Diputados) und den Senat (Cámara de Senados), aufgeteilt wurde (Art. $10 \mathrm{ff}$ der Verfassung von 1828). Der Präsident verlor jene, die übrigen Staatsfunktionen überragende Machtstellung und Amtsführung auf Lebenszeit, wie sie sich Bolívar in seinem Oktroi von 1826 zugewiesen hatte. Die Verfassung folgte dem Vorbild der nordamerikanischen Verfassung darin, dass der Präsident auf vier Jahre gewählt wurde und - in Abkehr von der entsprechenden Beschränkung des Jahres 1823 - eine einzige Wiederwahl unmittelbar nach Ablauf der Amtszeit möglich war (Art. 84). Ferner war er, anders als der König in der konstitutionellen Monarchie Spaniens im 19. Jahrhundert, dem Parlament gegenüber selbst für die Maßnahmen der Regierung verantwortlich (Art. 88), worin das Bestreben nach einer möglichst wirksamen Balance zwischen Exekutive und Legislative zum Ausdruck kommt.

Trotz des Misserfolgs hat Bolívars Konzept vermittelnder Gewalten im späten 19. Jahrhunderts doch noch als Vorbild gedient: Die kolumbianische Verfassung von 1886 verankerte die „Wählergewalt“ als weitere, vierte Gewalt neben den drei übrigen staatlichen Funktionen. Sie sollte vor allem aus Sicht ihrer konservativen Befürworter im Verfassungskonvent eine ausgleichende, vermittelnde Rolle im Institutionengefüge spielen. ${ }^{57}$

\section{d. Die Institution des „poder conservador" in Mexiko}

Mexiko geriet nach dem Ende des ersten Kaiserreiches unter Augustín de Iturbide (1823) in längere politische Turbulenzen, die im Grunde bis zur Präsidentschaft Porfirio Diáż(18771880 und 1884-1911) andauerten. Die Staatsführung wechselte zwischen konservativen und liberalen Regierungen, die weltanschaulich jeweils durch europäische Strömungen beeinflusst waren, wie sie vor allem in Frankreich und Spanien dominierten, nämlich entweder durch traditionelle, eher restaurative oder aber durch liberale Konzepte. Die erste republikanische Verfassung aus dem Jahre 1824 folgte in wesentlichen Punkten der zu dieser Zeit stark idealisierten nordamerikanischen Verfassung von 1787: Sie verankerte eine bundesstaatliche Ordnung, teilte die gesetzgebende Gewalt, den „Generalkongress“, in zwei Kammern auf (Abgeordnetenhaus und Senat, Art. 7) und entschied sich für die Konzentration der „obersten ausführenden Gewalt“ in der Person des Präsidenten (Art. 74). Er war damit unmittelbar Teil des gewaltenteiligen Systems, zumal er selber die Kabinettsmitglieder (Sekretäre) ernannte und entließ, außerdem im Gesetzgebungsverfahren über ein 
(suspensives) Veto verfügte (Art. 55 f). In seiner herausgehobenen Position sollte der Präsident aus Sicht der Konstituante eine gewisse Kontinuität mit der Monarchie verkörpern, wofür sich das große institutionelle Gewicht der spanischen Vizekönige im ehemaligen Neuspanien (Mexiko) anführen ließ. Zugleich sollte sich der Präsident in das System der Gewaltentrennung einfügen, was eigentlich einen Vorrang der Legislative hätte bedeuten müssen, insbesondere um einer Ausweitung der exekutiven Befugnisse zu einer autoritären Herrschaft oder gar einer Diktatur vorzubeugen. Stattdessen war es der Präsident, der in der Praxis ein starkes Übergewicht gewann ${ }^{58}$. Früh zeigte sich hier eine Tendenz, wie sie in der weiteren lateinamerikanischen Verfassungsgeschichte noch häufiger die Einführung des „reinen“ Präsidialsystems nordamerikanischen Musters begleitete: Der Grundsatz der Gewaltentrennung begünstigte jene übersteigerte Form persönlicher Herrschaft, die sich seitdem mit dem Begriff des „Caudillismo“ verbindet und die in ihrer Entwicklung durch die ausgedehnte ländliche Struktur, den niedrigen Bildungsstandard der Massen und das Fehlen einer Mittelschicht begünstigt wurde. ${ }^{59}$

Vor diesem Hintergrund ist die Aufhebung der mexikanischen Verfassung von 1824 und die Verkündung einer neuen Verfassung im Jahres $1836 \mathrm{zu}$ sehen, die eine konservative Handschrift trug und terminologisch an die spanische Rechtsgeschichte anknüpfte ${ }^{60}$. Mit bürgerlich-liberalen Konzepten, so dem Schutz der Individualrechte und dem Zensuswahlrecht, verbanden sich traditionelle Elemente, etwa der Schutz des katholischen Bekenntnisses und Anklänge an den bourbonischen Regalismus ${ }^{61}$. Um eine Balance zwischen Autorität und Freiheit zu gewährleisten, um einem drohenden Herrschaftsexzess ebenso entgegenzuwirken wie revolutionären Unruhen, sollte eine neue, vierte Gewalt als Garant der Verfassungsordnung fungieren und als Vermittler zwischen den übrigen drei Staatsfunktionen herausgehoben werden. Diese Rolle drückt die Bezeichnung „supremo poder conservador“ aus (2. Verfassungsgesetz), in Anlehnung an Benjamin Constants ,,pouvoir préservateur“. Das fünfköpfige Gremium besaß umfangreiche Befugnisse, so Gesetze und Verordnungen als verfassungswidrig aufzuheben, den Präsidenten abzusetzen, die Sitzungen des Kongresses zu suspendieren und Gerichtsurteile zu annullieren sowie ausnahmsweise, den ,nationalen Willen“ zu definieren, um revolutionären Bewegungen entgegenzuwirken (2. Verfassungsgesetz, Art. 12). Als verfassungshistorisch verdienstvoll darf der Versuch gelten,

Friedrich Wehner, Grundlagen einer mexikanischen Verfassungsgeschichte, Hamburg 1978, S. 122.

59 Karl Löwenstern, Der Staatspräsident. Eine rechtsvergleichende Studie, in: ders., Beiträge zur Staatssoziologie, Tübingen 1961, S. 340 f.

60

Die Bezeichnungen „Código de las Siete Leyes Constitucionales“ bzw. „Constitución de las Siete Leyes“ spielen auf „Las Siete Partidas“ an, die große Gesetzessammlung König Alfons X., des ,Weisen“(1252-1284). Otto Carlos Stoetzer, Benjamin Constant and the doctrinaire liberal influence in Hispanic America (Anm. 37), S. 28 f. 
erstmals eine tatsächliche Kontrolle zu institutionalisieren, um die hoheitliche Tätigkeit aller drei Staatsfunktionen auf ein verfassungsgemäßes Verhalten zu überprüfen. Auch wenn diesem Ansatz wenig Erfolg beschieden war, bereitete er die spätere (verfassungs)gerichtliche Kontrolle vor ${ }^{62}$. In der Praxis schwang sich jedoch der ,supremo poder conservador" zu einer Superrevisionsinstanz auf. Er maßte sich regelrecht diktatorische Vollmachten an und zwang den übrigen Funktionsträgern seinen Willen auf, betrieb also einen Missbrauch, den zu verhüten er selber bestimmt war. ${ }^{63}$

Die Verfassung von 1836 wurde bereits wenige Jahre später (1841) aufgehoben, auf den poder conservador als vermittelnde, neutrale Gewalt verzichtet. Die Ablösung der Verfassung von 1824 durch jene von 1836 belegt, dass die Einführung eines „reinen“ Präsidialsystems in Mexiko schon bald nach Gründung der Republik als unbefriedigend empfunden wurde. Deshalb suchte man nach Mischlösungen, die sich an europäischen Konzepten orientierten und zu Rechtsfiguren wie dem poder conservador führten. Obwohl Mexiko im Jahr 1846 zum Präsidialsystem nach dem (nordamerikanischen) Muster von 1824 zurückkehrte, griffen die Mexikaner auch in der Folgezeit wieder auf Vorbilder des europäischen Konstitutionalismus zurück, bis im Zweiten Kaiserreich (1863-1867) sogar ein erneuter Anlauf zur Monarchie unternommen wurde.

\section{Die Annäherung an den Parlamentarismus in Lateinamerika während des 20. Jahrhunderts}

Im 20. Jahrhundert entwickelte Lateinamerika verschiedene Gegenmodelle zu jenem Präsidentialismus, wie er auf das Beispiel der USA zurückgeht. Hier seien nur zwei behandelt, nämlich Uruguay und Kuba, weil sie besondere Bezüge zum Konzept Benjamin Constants aufweisen. Darüber hinaus verankerten weitere Staaten, wie etwa Chile (1891 und 1925), Regierungsformen, die sich dem Parlamentarischen System stark annäherten. Allen diesen Fällen gemeinsam ist die Suche nach Mischlösungen zwischen dem Präsidialsystem einerseits und dem (europäischen) Parlamentarismus andererseits.

\section{a. Uruguay: die Erweiterung der Gewaltenteilung im „colegiado integral“}

Auch die erste formale Verfassung Uruguays von 1830, die dann rund 90 Jahre gültig blieb, orientierte sich am nordamerikanischen Präsidialsystem: Der Präsident war als Staatsoberhaupt zugleich Chef der allgemeinen Verwaltung und konnte nur im Wege der Präsidentenanklage (,juicio público“), eingeleitet durch das Repräsentantenhaus, vor dem Senat zur 
Rechenschaft gezogen werden (Art. 26, 79). Einige Regelungen schwächten jedoch das Prinzip der Gewaltentrennung ab und zwangen den Präsidenten zur Kooperation mit dem Parlament: Der Präsident wurde weder direkt durch das Volk noch indirekt durch Wahlmänner gewählt, sondern durch beide Kammern gemeinsam in der sog. Allgemeinen Versammlung (Asamblea General, Art. 73). Jede der beiden Kammern konnte die Minister zur Befragung zitieren (sog. llamado a sala), wenn auch ohne das Tadelsvotum und die Kontrolle, wie sie mit einer ,interpelación“ verbunden gewesen wären ${ }^{64}$. Die Minister blieben für ihre Dekrete und Anweisungen voll verantwortlich (Art. 86) und waren nicht bloße Gehilfen oder Sekretäre des Präsidenten. Im Ergebnis entspricht bereits diese erste Verfassung einer abgeschwächten, gemäßigten Form des klassischen Präsidentialismus ${ }^{65}$; wenn nicht sogar bereits hier von einer „Mittelstellung“ zwischen dem ,reinen“ Präsidialsystem der USA und einem Parlamentarischen System gesprochen werden kann. ${ }^{66}$

Gegen Ende des 19. Jahrhunderts verstärkten sich die Reformbestrebungen. Der Präsident hatte, in Übereinstimmung mit den Tendenzen zum „Caudillismo“ in ganz Lateinamerika, seine persönliche Machtstellung erheblich gefestigt. Ganz abgesehen von seinen exekutiven Befugnissen, kontrollierte der Präsident die wichtigsten Wahlverfahren. Er wurde zur zentralen Figur der Wahlen, zum ,gran elector“, da er die für die Regionalwahlen verantwortlichen obersten Beamten auswählte und (außer seinen Ministern) in der Praxis auch seinen Nachfolger bestimmte ${ }^{67}$. Folgerichtig zielte die neue Verfassung von 1917 darauf ab, die Allmacht des Präsidenten einzudämmen, insofern sie dessen Befugnisse auf zwei Organe mit unterschiedlichen Geschäftsbereichen, aber ungefähr gleichem juristischen Gewicht aufteilte. Kern dieses „kollegialen Regierungssystems“ (colegiado integral) war eine „doppelköpfige Exekutive“ (ejecutivo bicéfalo): Dem Präsidenten, der vom Volk direkt gewählt wurde, unterstanden das Innen-, das Außen- und das Verteidigungsministerium. Neben ihm amtierte der ebenfalls durch das Volk gewählte Nationale Verwaltungsrat (Consejo Nacional de Administración), der den übrigen Ressorts vorstand. Im Ergebnis musste der Präsident seine Macht nicht nur mit dem Nationalen Verwaltungsrat teilen, sondern auch mit den Regierungsmitgliedern, die der ,doppelköpfigen Exekutive“ zugeordnet waren, außerdem mit den Autonomen Einheiten der Regionen (Entes Autónomos) sowie den Gemeinden, denen der dezentralisierte Verwaltungsaufbau einen nun größeren Handlungsspielraum einräumte. ${ }^{68}$

Ernst-J. Kerbusch, Das uruguayische Regierungssystem. Der Zweite Colegiado 1952-1967, Köln 1971, S. 15.

Joaquín Secco García, Síntesis de la evolución constitucional (1830-1966), in: ders., Constitución de la República Oriental del Uruguay, Montevideo 1982, S 5. 
Maßgeblichen Einfluss auf dieses Konzept übte José Batlle y Ordóñez (1856-1929) aus, Staatspräsident Uruguays von 1903 bis 1907 und 1911 bis 1915, der mit dem europäischen Verfassungsdenken seiner Zeit gut vertraut war. Der Grundgedanke folgt jenem Ansatz Benjamin Constants, der dafür eintrat, die ursprünglich einheitliche Position des Staatsund Regierungschefs in eine dritte und eine vierte Gewalt (oder Funktion) aufzuspalten, und damit letztlich den persönlichen Einfluss dieser neu gestalteten vierten Gewalt beschränkte, sie gleichsam ,neutralisierte“. Abweichend von diesem Konzept war allerdings innerhalb des „kollegialen Regierungssystems“ in Uruguay keine der beiden Teilfunktionen über die übrigen Funktionsträger herausgehoben, so dass sie eine vermittelnde, unparteiliche Rolle hätten ausüben können. Auch fehlte es an den charakteristischen Vorrechten eines ,pouvoir neutre“, etwa das Parlament aufzulösen oder die (übrigen) Inhaber der exekutiven Gewalt abzusetzen. Vielmehr war es das Parlament (der Kongress), das unter bestimmten Voraussetzungen die Rolle eines Schiedsrichters zwischen Präsident und Nationalem Verwaltungsrat spielte (Art. 18 Nr. 19). An die Stelle einer Trennung der Gewalten trat eine Verteilung der politischen Kontrolle, die trotz des komplizierten Systems bis in die dreißiger Jahre sehr zur politischen Stabilität des Landes beitrug. ${ }^{69}$

\section{b. $\quad$ Kuba: Der Präsident als ,poder moderador“}

Ein später, aber besonders enger Bezug zum Konzept des „pouvoir neutre“ findet sich in der kubanischen Verfassung von 1940. Sie stärkte die Position des Kongresses, um die politische Übermacht des Präsidenten einzudämmen. Dieser wird ganz im Sinne Benjamin Constants zur ,richtungsweisenden, mäßigenden und die Staatseinheit repräsentierenden Gewalt“ (Art. 138). Seine Position ist jedoch tatsächlich geschwächt, gleichsam „,neutralisiert", da er die Regierung gemeinsam mit dem Ministerrat führt. Wie im europäischen Parlamentarismus hat der Ministerrat auch hier die Funktion eines wirklichen Kabinetts, dem ein Ministerpräsident vorsteht (Art. 151 ff). Die Minister müssen sich dem Kongress gegenüber einzeln und als Gremium verantworten.

Die Besonderheit dieses Ansatz liegt darin, dass die Verfassung von 1940 versuchte, ein echtes Parlamentarisches System in das klassische Präsidialsystem einzubauen, weshalb man von einer ,halb“- bzw. ,,angenähert“-parlamentarischen Verfassung gesprochen hat ${ }^{70}$. Da der Parlamentarismus aus den konstitutionellen Monarchien des 19. Jahrhunderts hervorging, ist es plausibel das man in Kuba dem Staatsoberhaupt die Rolle eines ,poder moderador" übertrug und damit auf eine Figur rekurrierte, die in den Monarchien Spaniens und Frankreichs fester Bestandteil des juste-milieu-Denkens gewesen war. Diesen ideengeschichtlichen Rekurs dürfte der Umstand erleichtert haben, dass Kuba besonders lange zum 
spanischen Königreich gehörte (bis 1898) und daher durch spanisch-europäische Staatslehren beeinflusst blieb.

\section{Ergebnis}

Ebenso alt wie die Verfassungsgeschichte der lateinamerikanischen Republiken, ganz abgesehen von den konstitutionell-monarchischen Ansätzen, ist auch die Suche nach Mischformen. Diese milderten das Prinzip der Gewaltentrennung ab und damit jenes „reine“ Präsidialsystem, in dem der vom Volk direkt gewählte Präsident alleiniger Inhaber der ausführenden Gewalt (Funktion) ist, und die Minister lediglich seine Gehilfen und Berater sind, welche ohne Rücksicht auf die Mehrheitsverhältnisse durch ihn ernannt und entlassen werden und rechtlich gesehen selbst dann nicht an der Exekutive teilhaben, wenn sie seine Amtshandlungen gegenzeichnen müssen. Soweit jedoch die lateinamerikanischen Verfassungen des 19. Jahrhunderts auf Benjamin Constants Lehre und deren Anwendung in Frankreich und Spanien zurückgriffen, um die klassische Dreiteilung der öffentlichen Gewalten (oder Funktionen) zu erweitern, wichen sie wiederholt von einem seiner wesentlichen Grundgedanken ab: So waren sowohl in der monarchischen Verfassung Brasiliens (1824) als auch im republikanischen Konzept Simón Bolívars (Bolivien und Peru 1826) die Befugnisse des Staats- und Regierungschefs umfassender bemessen, als sie nach Benjamin Constant dem ,pouvoir neutre“ zustanden. Weder war hier von einer Trennung der Autorität des Staatsoberhauptes von der eigentlichen Regierungsgewalt zu sprechen noch vom erwähnten juste-milieu-Ideal eines Bürgerkönigs, der unter den Bedingungen des Parlamentarismus ,herrschte, aber nicht regierte“. Konsequenterweise betraute Bolívar andere Institutionen als den Staatschef mit einer vermittelnden, im Grunde geschwächten Funktion, als er eine „Wählergewalt“ und eine „Moralische Gewalt“ schuf.

In der 1. Hälfte des 20. Jahrhunderts sind Uruguay und Kuba Beispiele für eine weit verbreitete Tendenz in Lateinamerika: Ausschlaggebend für die Suche nach Mischlösungen zwischen dem Präsidial- und dem Parlamentarischen System war die Erfahrung oder die Befürchtung, dass der „klassische“ Präsidentialismus nordamerikanischer Herkunft zu einem Machtmissbrauch durch den Staats- und Regierungschef führte. Jedoch ist jeweils schwer zu entscheiden, welche Elemente in solchen ,gemischten Verfassungen“ überwiegen, da dies oft weniger vom Verfassungstext selber als vielmehr von parteipolitischen Konstellationen, der innen- und außenpolitischen Lage des Landes sowie der Person des Präsidenten abhängt ${ }^{71}$. Was die Rolle der ,,vermittelnden“ oder „,neutralen Gewalt“ anbelangt, so ging diese in Uruguay eher auf das Parlament über. Hierin liegt ein gewisse Parallele zur älteren Konzeption Simón Bolívars in Bolivien und Peru, der hiermit allerdings ganz anders als im Falle Uruguays die Schwächung der Legislative und eine weitere

Ernst J. Kerbusch, Das uruguayische Regierungssystem (Anm. 64), S. 7 f. 
Stärkung der Exekutive bezweckte und deshalb neue, miteinander konkurrierende Gremien schuf. Einen späten, aber besonders engen Bezug zum Konzept des ,,pouvoir neutre“ stellte die kubanische Verfassung von 1940 her. Ganz im Sinne Benjamin Constants wird das Staatsoberhaupt zur „mäßigenden und die Staatseinheit repräsentierenden Gewalt“. Seinen tatsächlich geschwächten Befugnissen entspricht die stärkere Position des Kongresses, der in die Lage versetzt werden soll, die politische Übermacht des Präsidenten einzudämmen. 